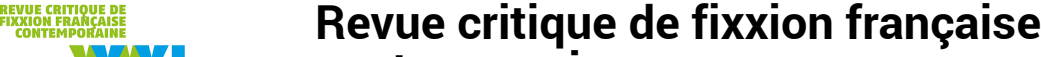 \\ WXI contemporaine
}

\section{Le roman noir français du XXI ${ }^{\mathrm{e}}$ siècle, espace du nouveau désordre mondial et des rapports de méfiance radicale}

\section{Stéphane Ledien}

\section{OpenEdition}

Journals

Édition électronique

URL : https://journals.openedition.org/fixxion/553

DOI : $10.4000 /$ fixxion.553

ISSN : 2295-9106

Éditeur

Ghent University

\section{Référence électronique}

Stéphane Ledien, «Le roman noir français du XXIe siècle, espace du nouveau désordre mondial et des rapports de méfiance radicale », Revue critique de fixxion française contemporaine [En ligne], 20 | 2020, mis en ligne le 15 juin 2020, consulté le 16 mai 2022. URL : http://journals.openedition.org/fixxion/553 ; DOI : https://doi.org/10.4000/fixxion.553

Ce document a été généré automatiquement le 16 mai 2022.

\section{c) (i) () $\Theta$}

Les contenus de la Revue critique de fixxion française contemporaine sont mis à disposition selon les termes de la licence Creative Commons Attribution - Pas d'Utilisation Commerciale - Pas de Modification 4.0 International. 


\title{
Le roman noir français du XXI siècle, espace du nouveau désordre mondial et des rapports de méfiance radicale
}

\author{
Stéphane Ledien
}

De par leurs récits oscillant entre un détachement quelque peu ludique et un nihilisme radical, les romans noirs français du $3^{\mathrm{e}}$ millénaire apparaissent comme un point de non-retour à l'intérieur du domaine de la littérature policière. Certes, les discours de transgression que porte le genre actuel pourraient s'inscrire dans la droite ligne d'un "néo-polar"1 où se croisaient déjà "la critique des formes les plus extrêmes de la politique, et la déconstruction des mécanismes de la domination sociale et de nos perceptions du bien et du mal"'. Symptomatique de la crise aggravée par le premier choc pétrolier, le néo-polar était mis en œuvre par des écrivains en marge, souvent militants et revendiqués défenseurs des classes laborieuses ${ }^{3}$. "Dégauchisé", le noir hexagonal d'aujourd'hui se caractérise par une narration moins polarisée qui joue à cache-cache avec les bon sens moral et politique : probité et déloyauté, souffrance et contentement s'y fondent et s'y confondent de manière troublante. Il est ainsi devenu la caisse de résonance d'auteurs désireux de "partir en guerre contre [le] réel étouffant, lui faire son compte artistiquement" 4 : sa violence confine ici et là à l'ultime réaction d'une volonté de préférence du néant articulée à une apparente absence de sens de la vie, ainsi qu'à l'inexistence de morale et de vérité. Indéniablement, une telle radicalisation concourt à l'affirmation de formes plus conflictuelles que jamais, à travers lesquelles les narrateurs paraissent à la fois s'engager et se désengager à l'égard de toute magnanimité et de leurs personnages, voire, de leur propre énonciation. 


\section{Rupture ou révolution?}

2 À l'aube de notre deuxième décennie, le critique Baptiste Liger présentait dans la revue Lire ce qui lui semblait constituer la relève du polar engagé à la française, qu'il appelait alors le "NPN" : le "Nouveau Polar Nihiliste". "Nouveau", précisait-il,

en ce qu'il témoigne d'un fort changement esthétique et [...] politique ; polar, car tous les codes du genre sont honorés ; Nihiliste, parce que la vision du monde de tous ces jeunes loups correspond d'assez près à la définition du Larousse : Négation des valeurs intellectuelles et morales communes à un groupe social, refus de l'idéal collectif de ce groupe. ${ }^{5}$

3 Liger se référait notamment aux auteurs DOA, Antoine Chainas et Jérôme Leroy. Le portrait qu'il dressait de ces signataires de "polars asociaux aux antipodes des revendications de la génération Pouy-Daeninckx-Raynal" autorise cependant d'élargir le champ à d'autres plumes versées dans la "peinture désenchantée du monde" ${ }^{6}$. En l'occurrence celle de Dominique Manotti, qui a d'ailleurs coécrit avec DOA L'honorable société en 2011, après une fiction remettant en jeu les bavures policières, Bien connu des services de police ${ }^{8}$. Et celle d'Elsa Marpeau, dont la production s'est accélérée depuis la publication en 2010 de son deuxième opus, Les yeux des morts ${ }^{9}$. Sans constituer une école officiell $^{10}$, ces romans posent de nouvelles questions politiques débarrassées des oppositions et de l'idéalisme social de leurs prédécesseurs imprégnés

d'une idéologie de gauche ou d'extrême gauche, dans l'ensemble très marqués par le marxisme [...] et par la recherche dans l'histoire du $\mathrm{XX}^{e}$ siècle français de résonances pouvant expliquer l'échec de Mai $68 .{ }^{11}$

Selon Luc Boltanski, le roman policier a très tôt représenté la contradiction entre "l'État [qui met] en place un ordre [...] sur un territoire enfermé dans des frontières" et "le capitalisme [qui] se joue" ${ }^{12}$ de celles-ci. Le polar a orchestré l'appréhension suscitée par cette opposition, et cette inquiétude a joué un rôle

très important au tournant $\mathrm{du} \mathrm{XIX}^{\mathrm{e}}$ et du XXe siècle, c'est-à-dire précisément au moment où se [sont] développ[é], d'un côté, le capitalisme en pleine expansion et, de l'autre, les prétentions de l'État-nation à tout régir ${ }^{13}$.

Or, l'effondrement du communisme et "des systèmes de pensée qui gravitaient autour" ${ }^{14}$, l'après-11 Septembre, les crises financières et migratoires, le terrorisme islamiste, les résurgences nationalistes dans certaines régions du globe ou encore, les échecs de l'intégration française et du fonctionnement démocratique de l'Union européenne, ont bouleversé la donne axiologique. Plus qu'une rupture, le XXI siècle a engendré la nécessité d'une réinvention générique, pour aboutir, comme l'a énoncé Dominique Manotti, au "roman noir de la mondialisation, du capitalisme triomphant sans adversaire structuré, sans limites" ${ }^{15}$. Soit un contenu réfractaire à la tradition, qui ne place pas toujours ses narrateurs dans le camp de ceux qui pensent bien et, à moins d'en pervertir l'utopie, ne défend plus forcément "le pauvre, le faible, la racaille"16.

\section{Une littérature des rapports de méfiance}

Marqués par cette nouvelle ère du soupçon, les romans concernés optent pour une peinture à fonction évaluative des rapports de méfiance entre différentes figures d'autorité ou de contre-pouvoir, ainsi qu'entre le narrateur et ses personnages. Dans le premier cas, des protagonistes représentant la police, l'armée, le gouvernement ou un 
service de renseignement se disputent une influence quelconque, tandis qu'à un échelon moindre ou dans la clandestinité, d'autres figures cherchent à se libérer des "dominants" persécuteurs ou à renverser le pouvoir.

Dans Black Blocs ${ }^{17}$ d'Elsa Marpeau, une post-doctorante en physique prénommée Swann retrouve chez elle le corps sans vie de son compagnon Samuel, professeur de sociologie. Après qu'elle a découvert que son conjoint était aussi le leader d'un groupe d'anarchistes autonomistes, Swann commence à côtoyer le groupuscule et comprend qu'il prépare un acte de sabotage. Le conflit de loyauté qu'elle éprouve est alors appuyé par une narration tiraillée entre monstration politique des coups de force et gratuité de tout accès de violence. Entre compréhension du camp étatique et dangereuse attraction pour celui des militants, le dilemme de Swann atteint son apogée lorsqu'elle apprend par des membres de services de contre-sécurité intérieure que Samuel agissait comme informateur. Au manichéisme manifeste, le récit préfère une ambiguïté indébrouillable : le final montre le monde de Swann sur le point de peut-être basculer dans une guerre totale contre l'État et toute forme d'ordonnancement.

8 La même oscillation prévaut dans L'honorable société, signé à quatre mains par DOA et Dominique Manotti, où des militants écologistes qui ont piraté l'ordinateur du directeur de la sécurité du Commissariat à l'énergie atomique assistent, par webcam interposée, au meurtre de celui-ci. Préférant disparaître dans la nature, les activistes deviennent de parfaits coupables, assimilés par la presse à des écoterroristes membres de "Black Blocs". Dans le même temps, une partie de la classe politique, en particulier l'équipe de campagne d'un ministre candidat aux élections présidentielles, cherche à dissimuler ses liens avec cette ténébreuse affaire. Si une forme de maintien éthique se retrouve dans la figure du commandant de police chargé de l'enquête, le lecteur éprouve la sensation d'être ballotté d'une raison d'État, et d'une politique d'action directe, à une autre. Quoiqu'elle pointe les meurtres perpétrés illégalement par des agents du renseignement intérieur, la narration capte aussi l'inconscience et la simplicité idéologique des activistes incapables de s'évanouir dans la nature - celle-là même pour laquelle ils prétendent se battre.

Dans Bien connu des services de police de Dominique Manotti, des agents de la brigade anti-criminalité d'un commissariat de la région parisienne se livrent à toutes sortes de comportements déviants (extorsions de prostituées, contrôles d'identité qui dégénèrent et entraînent la mort d'un jeune issu de l'immigration) au cœur d'une banlieue de l'est francilien menacée d'explosion sociale. Pendant que des "bavures" ont lieu ici et là à Panteuil, Le Muir, la commissaire locale dévorée par l'ambition, perpétue une politique de maintien de l'ordre radicale qui sert les objectifs du ministre de l'Intérieur. On y relate également le parcours difficile de jeunes recrues confrontées, d'une part à la violence aussi bien à l'intérieur qu'à l'extérieur de leur commissariat, d'autre part au racisme quotidien de la police dans les quartiers défavorisés. En contrepoint, l'intrigue suit l'enquête menée par Noria Ghozali, commandante aux Renseignements Généraux. Celle-ci cherche à percer le secret des contacts noués entre policiers du secteur et certains grands voyous, tout en espérant, avec le soutien d'un ancien du service, faire tomber l'arriviste Le Muir. La probité qu'incarne Ghozali aux yeux du lecteur n'équilibre cependant qu'à peine les forces délétères en présence : si l'enceinte policière (au propre et au figuré) devient le siège des pires exactions, où le citoyen peut-il se sentir en sécurité ? Opposition constante des bévues autoritaires, des malversations politiques et d'un mal soigné par le mal (brutalité policière contre 
violence des quartiers dégradés, maintien de l'ordre politique par l'exacerbation du désordre communautaire...), le récit de Bien connu des services de police s'évase dans l'irrésolution sociale et l'impossibilité d'une justice équitable.

10 Exploration fictive des arcanes du pouvoir, cette fois-ci du côté des partis politiques, $L e$ $B l o c^{18}$ de Jérôme Leroy a pour toile de fond une "nuit des longs couteaux" de l'extrêmedroite française. Alors que la situation s'est détériorée sur l'ensemble du territoire, le Bloc Patriotique (calque du Front National) apparaît au gouvernement de droite comme un "ultime recours" d'autorité. Pendant que des négociations ont lieu dans les palais du pouvoir pour savoir combien de ministères vont revenir au Bloc, deux hommes "de l'ombre" attendent, chacun cloîtré dans une chambre d'hôtel. D'un côté, Antoine Maynard, mari de la présidente du parti, relate au "tu" (procédé forçant l'empathie du lecteur) son ascension et comment il a trahi son "camarade" Stanko. De l'autre, Stanko, ancien skinhead et exécuteur des basses œuvres du parti, se prépare, mi-fataliste, mirévolté dans une narration homodiégétique teintée de mélancolie et de violence, à recevoir les tueurs envoyés à ses trousses. La frontière qui sépare les hommes de main des apparatchiks s'avère poreuse. Le récit joue de cette fluctuation en déconstruisant une vieille amitié politique et en la reconfigurant en rupture et en confrontations esthétique et actantielle.

11 On retrouve le personnage de Stanko et des protagonistes du Bloc dans L'ange gardien ${ }^{19}$. Le roman met en scène trois personnages : le tueur Berthet, soudain en disgrâce avec la cellule secrète (l'Unité) qui l'a toujours employé ; l'écrivain Martin Joubert, exenseignant dans des quartiers difficiles devenu poète et auteur de polars, que Berthet veut charger d'écrire le récit de sa vie de barbouze ; et Kardiatou Diop, jeune ministre noire icône de l'intégration dont Berthet est secrètement amoureux et qu'il veut protéger de menaces complotistes. Évidemment, ces personnages n'agissent pas toujours en vertu des idéologies qu'ils incarnent. Droiture politique et sédition, modération et violence, raffinement et rudesse s'amalgament à travers les points de vue du tueur mandaté par un organisme à l'obédience politique indéchiffrable, de l'écrivain esthète fasciné par la brutalité, et du jeune politicien adepte, malgré son progressisme, de la manipulation médiatique.

Dans son roman Pur $^{20}$, Antoine Chainas remet aussi en scène, avec un traitement dystopique, le climat d'insécurité, les idéologies extrêmes et la crise de l'opinion qu'ont amenés l'Europe et son abolition des frontières, la montée conjointe de l'islamisme et des nationalismes. Pur raconte comment, dans le Sud de la France et un avenir potentiellement proche, un certain Patrick Martin, qui a perdu sa femme au cours d'un accident de la route, bascule dans la violence raciste, la paranoïa et les représailles aveugles. Le personnage croit se rappeler que deux Arabes ont tiré sur son véhicule tandis que sa femme et lui roulaient sur l'autoroute. Un capitaine obèse qui enquête sur les meurtres d'automobilistes d'origine maghrébine le soupçonne cependant de dissimuler des informations. Le récit revient aussi sur les pérégrinations existentielles d'un adolescent sous l'emprise de son père prédicateur et psychopathe, et des communautés vivant repliées dans des résidences encadrées par des milices privées. Antoine Chainas aborde des thèmes chers à sa génération d'écrivains de noir : collusion entre les politiques et la police, fracture majeure au sein de la communauté française. Le maître-mot serait peut-être aussi celui du tabou : tabou de la peur de "l'étranger", tabou du préjugé, du sexe, de la laideur (difformité du policier obèse) ou de la couleur de peau qu'on n'ose pas relever tout en la voyant, tabou des "mauvaises pensées 
politiques". À ce sujet, Chainas avait déjà montré qu'il refusait "toute leçon de morale" ${ }^{21}$ en déléguant la narration à un policier noir dealer dans Anaisthêsia ${ }^{22}$ : écrit sur un ton tantôt ironique, tantôt clinique, voire morbide, le roman met en scène le sergent-détective Désiré Saint-Pierre, personnage trouble qui, à la suite d'un accident de voiture l'ayant défiguré, se retrouve frappé d'une anomalie neurologique qui le rend indifférent à la douleur. Outre ses états d'âme caractérisés par un manque total d'empathie, Saint-Pierre relate sa tentative d'arrêter une meurtrière surnommée "la Tueuse aux Bagues", mais aussi de découvrir ce qu'il est advenu de la cargaison de drogue dont il avait la charge. "Flic noir dans une ville blanche" ${ }^{23}$ gangrénée par le crime et le racisme, Saint-Pierre mène le lecteur par le bout du nez, jusqu'à des révélations nauséeuses qui tranchent avec l'héroïsme dont on croyait le personnage investi, et un dénouement punitif insoupçonné. Comme Leroy plongeant le lecteur dans la tête d'un fasciste violent mais au lyrisme fascinant, Chainas joue avec la fin des illusions de la rectitude politique.

Et ils oublieront la colère ${ }^{24}$, d'Elsa Marpeau, manie lui aussi un tabou toujours vivace de la société française : celui de la collaboration. Le récit entremêle deux temporalités dans une même région rurale de la France (l'Yonne) : la période de la Libération, soit en 1944, et l'époque contemporaine, en 2015. Dans la première, il est question de Marianne Marceau, jeune femme en rupture avec les valeurs de son milieu et soupçonnée, pendant la fin de l'occupation, de "collaboration horizontale". Dans la deuxième, la capitaine de gendarmerie Garance Calderon enquête sur le meurtre d'un instituteur qui écrivait un essai sur les femmes tondues lors de la Libération. En retraçant l'histoire des Marceau, la gendarme va mettre au jour une troublante affaire d'inceste, d'usurpation identitaire et de xénophobie bien enracinée dans certaines campagnes de l'Hexagone. Là encore, dès que la narration s'occupe d'ausculter les travers d'une communauté, elle ne peut s'empêcher d'apporter une nuance de poésie (poèmes qui jalonnent aussi à proprement parler, par des emprunts et citations, Le Bloc et L'ange gardien), avec un champ lexical de l'art pictural et force métaphores :

Devant un cadavre, elle se pose en observatrice, comme devant une toile de maître. Il y a des tableaux tourmentés, agressifs, jouant sur des gammes chromatiques contrastées ; d'autres sont colorés, presque joyeux ; certains sont tranchés, noir et blanc ; d'autres, monochromes. Certains n'usent que des couleurs primaires. Garance en a même vu qui avaient la douceur mélancolique de la sépia. ( $\left.C_{0}: 20\right)$ D'abord l'image d'ensemble : le bois, l'herbe et l'eau. Dans cette composition estivale, le cadavre n'est qu'un détail de l'ensemble. Puis la technicienne se rapproche, saisit le mort allongé dans son écrin de verdure, avant de le fractionner en multiples pièces éparses. [... ] En contemplant le corps étendu, sa peau brune et sa posture gracieuse, [Garance] pense à des pays, des paysages, des musiques, d'autres visages, d'autres corps. (Co:25)

Par un mouvement de sublimation, la dimension sensorielle du texte prend des allures de complicité voyeuse. Plus encore, cette façon de réintégrer un cadavre dans une composition esthétique à outrance - phénomène qui rejoint par exemple l'idéalisme littéraire de Joubert perverti par l'“écriture alimentaire" de fictions Mommy Porn dans L'ange gardien - peut être lue comme un retour extrême à la primitivité du réel et au besoin paradoxal de s'en protéger. La brutalité ne va pas sans une indicible beauté. Le Beau peut constituer le dernier rempart face à un profond désenchantement, il n'en est pour autant plus subordonné au bien.

Avec Or noir ${ }^{25}$, Dominique Manotti immerge le lecteur et son personnage d'enquêteur homosexuel, le commissaire Daquin ${ }^{26}$, dans une décadence similaire. L'histoire se situe 
à Marseille dans les années 1970, ville ensanglantée par les règlements de compte liés à la French Connection, ainsi que par les conflits entre divers services de police et la prolifération de réseaux semi-clandestins (la police parallèle du Service d'action civique, la franc-maçonnerie...). Daquin cherche à élucider l'assassinat d'un ancien caïd de la drogue et de son associé autrefois membre des services secrets reconvertis dans les affaires pétrolières. Dans l'ombre, l'on assiste aussi à l'ascension fulgurante de traders que rien n'arrête, requins plus dangereux encore que les seuls gangsters auxquels le policier pensait avoir affaire. En plus de l'idée d'une impossible justice à rendre au final, le constat de la violence et de l'indécence qui règnent dans ces milieux d'influence se trouve sans cesse contrebalancé, sans doute pour mieux piquer au vif le sujet, par la vision délicate de Daquin, amoureux des plaisirs de la table et de la chair. Par le jeu des focalisations, le lecteur se laisse entraîner dans les tentations du personnage. Le narrateur oppose ainsi souvent, mais en brouillant avec subtilité les pistes axiologiques, finesse et vulgarité, élégante retenue et décharge de brutalité ou de sexualité.

De manipulations, il en est aussi question à l'échelle planétaire et à l'aune d'une rupture éthique totale dans la saga Pukhtu de $\mathrm{DOA}^{27}$. Le premier volume, Pukhtu primo ${ }^{28}$, suit principalement les membres de la clandestine unité paramilitaire $6 \mathrm{~N}$ dirigée par un certain "Voodoo" et œuvrant en Afghanistan pour le compte de la CIA. Ces combattants armés au caractère barbouzard deviennent à la fois proies et chasseurs d'un chef de guerre pachtoune, Sher Ali Khan Zadran, dont la famille a été décimée par la frappe d'un drone américain. Ultraviolence des rapports, ambiguïté axiologique et dissolution des repères moraux à l'appui d'une narration au "réalisme poussé" 29 déterminent un récit radical : avec son énonciation musclée du mercenariat, des dessous viciés du pouvoir et de meurtres et de viols de femmes et d'enfants, le monde crépusculaire de Pukhtu primo se complaît dans l'impossibilité d'un apaisement, aussi bien moral que politique.

\section{Confrontations, distanciations ironiques}

17 Derrière la violence des rapports mis en scène, ces romans construisent une double ironie, celle du sort et des relations entre les personnages. La première se manifeste notamment par une raillerie à l'égard du narrateur ou des protagonistes. Dans Le Bloc, Stanko l'ancien skinhead relate ainsi :

Je m'étire, je me regarde dans la glace au-dessus du lavabo. Cernes, rides creusées, la calvitie qui gagne comme deux langues de désertification sur chaque côté du crâne. J'ai joué au skin pendant deux ans et maintenant je pleure parce que je perds mes cheveux. (LB: 143)

Dans L'ange gardien, le narrateur hétérodiégétique énonce à propos du tueur Berthet : "Berthet se refuse à admettre que Berthet est essoufflé" ( $A G$ : 90). À propos de l'écrivain Martin Joubert, il ajoute : "Martin Joubert est soulagé même si Martin Joubert ne va pas bien" (AG : 144). Et dans Anaisthêsia, Saint-Pierre se laisse aller au commentaire suivant lorsqu'il croise des jeunes en sortant de chez lui : "Des Noirs, comme moi, qui, plus grands, feront des enculés, comme moi” (An:24). Même lorsqu'il est homodiégétisé, le narrateur semble capable de se renier, se désolidariser de sa condition magnanime. On perçoit, au fil de ces lectures, une ironie hermétique niant toute forme d'éthique de l'énonciation: le lecteur constate l'immoralité littéraire du narrateur, qui non seulement va parfois jusqu'à se moquer de lui-même ou de ses destinataires, mais 
n'hésite pas non plus à se contredire, se récuser, se complaire dans l'imprécision ou le leurre de ses propres perceptions. Dans Anaisthêsia encore, lorsque Saint-Pierre (qui habite un quartier malfamé) se rend à son appartement, il relève ainsi : "J'enjambe des corps allongés [...]. Endormis ou morts, ça n'a pas de réelle importance" (An: 61).

19 L'étendue des tonalités ironiques des textes constitue un vecteur de stylisation de la méfiance qu'éprouvent les personnages les uns à l'égard des autres : “Une fraction de seconde, Garance se demande si Isabelle est mentalement retardée ou si, au contraire, elle plane au-dessus de la mêlée dans une indifférence [...] sublime" (Co:30).

La même défiance, doublée de soupçons du narrateur envers ses personnages, s'illustre dans cet extrait de L'honorable société, où la brigade criminelle débarque sur une scène de crime d'abord prise en charge par le commissariat du secteur :

Dans la police comme ailleurs, l'implacable réalité de la chaîne alimentaire est à l'œuvre. Un crime, c'est pour la Crim'. Surtout si elle n'a pas grand-chose à foutre en ce moment. "C'est sa compagne qui nous a appelés. Vers deux heures du matin. [...]". Là, il s'adresse à l'homme juste derrière lui, le plus affable des trois. Petite quarantaine, pas très grand, tignasse brune, raie de côté, lunettes. [...] Commandant Michel Pereira, Criminelle, c'est comme cela qu'il s'est présenté. Commandant. Et c'est lui qui parle le plus. Sans doute le chef de groupe. (HS : 24)

21 Le lecteur apprendra, au fil de répliques autoritaires, que Pereira n'est pas le chef. À l'instar de l'incapacité de la gendarme Garance à caractériser la véritable nature de celle qu'elle interroge, le regard que jette le narrateur sur le commandant Pereira s'avère biaisé, miné par l'incrédulité.

La position des narrateurs donne, de fait, l'impression d'être aussi instable qu'ambiguë, et parfois réticente. Il n'est pas rare, alors, que l'instance de narration balaie toute objection de savoir du lecteur:

Berthet resta un long moment à traîner devant les tables et les rayons en songeant à tout ça, et à d'autres choses que son âme tourmentée charriait à flots continus mais qui ne nous intéressent pas pour l'instant. (AG:62)

23 Ailleurs, la réalité décrite par les personnages-narrateurs va même jusqu'à revêtir une dimension hypothétique : "Des ombres, dans les ruelles aveugles de part et d'autre de l'avenue, se glissent, furtives. Hommes ou animaux, je ne sais pas." (An: 61)

En compromettant ouvertement la crédibilité de leurs voix narratives et en amenant le lecteur à douter du bien-fondé de la confiance qu'il est censé accorder à ces instances, les textes flirtent avec le concept de "narrateur non fiable" tel que théorisé par Wayne C. Booth en $1961^{30}$. La non-fiabilité en présence ne saurait pourtant se définir par comparaison avec les normes et les valeurs de l'auteur implicite, puisque dans de nombreux cas, ce dernier se refuse à les communiquer ${ }^{31}$. Doit-elle alors s'évaluer, comme l'a proposé Frank Wagner, "avec un ensemble de connaissances préexistantes $\mathrm{du}$ monde et de la littérature que possèdent lecteurs et critiques" ${ }^{32}$ du genre ? Si tel est le cas, l'ambiguïté n'en reste pas moins présente en cette époque de globalisation. À la lueur des indices textuels de non-fiabilité factuelle et/ou idéologique déjà repérés, la question apparait plutôt susceptible "d'entrer en phase avec [des] réflexions sur la mystification [...], la manipulation [...], la mauvaise foi [...] ou encore l'imposture [...]" ${ }^{33}$.

25 Cette démarche, au fond, prolonge les mécanismes de distanciation ironique des romans de Manchette, qui selon le théoricien Franck Frommer, "mett[aient] en œuvre une tension [...] entre engagement et retrait, perte des valeurs et recomposition axiologique, défaite de la morale et reconstruction politique" ${ }^{34}$. Le phénomène est ici 
poussé à son paroxysme : chaque scrutation, chaque échange de regards renvoie à un vacillement entre la lucidité extrême et la duplicité ambiguë de la figure du narrateur, qu'il s'agisse de dresser un tableau historique ou un contexte d'intervention des personnages.

On ne peut pas dire qu'ils n'avaient pas annoncé la couleur, et bien avant la Libération. C'était si simple, il leur suffisait d'une tondeuse ou d'une paire de ciseaux. De toute façon, les cimetières étaient pleins. ( $\left.C_{0}: 34\right)$

Amina ne lui fait pas remarquer qu'il pourrait se servir de son ordinateur pour ça, ou d'un de leurs deux Smartphones. Amina le pense peut-être. Ou peut-être pas. Amina prend ce qui vient. Amina pourrait être une femme idéale. Même s'il n'y a pas de femme idéale. $(A G: 6)$

Lorsqu'il tient à démontrer son acuité, le narrateur ne peut s'empêcher de glisser vers une déloyauté à l'égard de son personnage - entité qu'il dessaisit de son autorité - et du lecteur. Toute volonté de dénonciation se dissout, du même coup, dans les impostures et les illusions déployées pour parer à l'irrésolution. Aucune voix n'accorde semble-t-il sa confiance d'action et d'énonciation aux autres locuteurs et/ou destinataires.

\section{Rapprochements, désillusions et opacité du réel}

Force, aussi, est de constater que les œuvres en présence multiplient les jeux de miroirs et les constructions labyrinthiques ou métatextuelles sur le rapport du protagoniste à la littérature et au polar. En plus d'importantes apostrophes directes au lecteur, ces procédés favorisent le "jeu réflexif d'un moi critique comme le théâtre dans le théâtre [...] qui détruit [...] l'illusion et instaur[e] un second degré" ${ }^{35}$ : les narrations au "tu" du Bloc (chapitres énoncés par Antoine Maynard) et de L'ange gardien (troisième partie, dévolue à Kardiatou Diop), sans compter toutes les fois où l'un des protagonistes se scrute dans la glace, agissent comme réflecteurs ludiques. De même, les mentions d'un personnage lisant (voire écrivant) un polar, ici et là dans Bien connu des services de police, L'ange gardien ou encore L'honorable société, participent à une évidente mise en abyme du genre, clin d'œil à l'acte de lecture en cours. Quelques exemples l'ont déjà démontré, les romans concernés n'excluent pas non plus, à l'instar de la génération d'auteurs précédente dont Jean-Bernard Pouy incarne la persistance, une forme acerbe d'humour noir. On retient cependant plus encore la caractérisation décalée et anti-héroïque dont ces romans font preuve et, point crucial, les comparaisons et métaphores incongrues auxquelles ils s'adonnent dans des passages d'une grande tension ou d'une rare violence. À preuve, cette scène de décapitation dans Pukhtu primo :

L'acier dérape sur l'os, grince, fouille, il veut un interstice sur lequel s'appuyer, par

lequel passer. [...] Ça poisse, ça glisse, [Sher Ali] est trahi par son propre sang. [...] Une fente, le poignard trouve, s'enfonce, taille. Bouche grande ouverte sur une langue mâchée de terreur, la tête refuse cependant de se détacher. (PP : 203)

Comme pour subvertir l'horreur de la scène, le narrateur ajoute ces quelques mots, acmé d'une violence absurde illustrée par une comparaison saugrenue : "Sher Ali se met alors à la dévisser des deux mains, à la manière d'un bouchon" (PP : 204).

Sans doute rejoint-on avec ce type d'incongruité ce que Pierre Schoentjes décrit comme un phénomène de distanciation critique, sorte de "légèreté [...] qui pousse l'artiste [le narrateur ?] à sourire de sa propre création" ${ }^{36}$. Le sourire, ici, s'efface au profit d'un rictus signifiant l'écœurement voire, le cynisme, à l'appui d'observations mimordantes, mi-désabusées et teintées d'irrévérence de la part du narrateur anonyme. 
30 Littérature de tensions, le roman noir s'affirme via ces exemples comme un contenu de friction des sens et de mise en opposition des intentions. Il n'est pas rare d'y déceler des principes d'auto-ironie (façon de se mettre en question à laquelle les narrateurs du Bloc et de L'ange gardien ont souvent recours), et, par-delà les collusions entre personnages, une connivence embarrassée entre le narrateur et ses protagonistes les plus vils, voire, entre le narrateur et lui-même (reflet dans lequel se projette parfois le lecteur). Ces accointances illustrent bien de façon transversale les rapports de méfiance dont il est question sur le plan de l'intrigue comme en matière de langage et d'énonciation :

Et c'est un match qui oppose entre elles des basketteuses sud-coréennes sur lequel tu tombes. Elles ont des moues concentrées dans l'effort et les cheveux noirs collés par la sueur sur les tempes comme dans ces films pornographiques asiatiques, vaguement sado-maso-bondage, que tu as appréciés, sois honnête, à une certaine période de ta vie. Tu affectes de te demander ce qui t’a réveillé, mais [...] tu ne le sais que trop bien. (LB:44)

31 À travers leurs scènes et dialogues d'affrontement ainsi que leurs descriptions déchirées entre des sensations contradictoires (sublime / grotesque, satisfaction / dégoût), les textes en présence cherchent à situer un ou plusieurs de leurs personnages, et au final leurs narrateurs eux-mêmes, en situation de critique du réel. Tous, comme le relève Luc Boltanski à propos des individus engagés dans des enquêtes et "événements",

ne peuvent prendre position qu'en formant un jugement sur la valeur de vérité de ce qui leur est donné pour réel. Or ce jugement n'a d'autre support que la crédibilité qu'ils accordent aux instances transmettant les informations, crédibilité qui dépend elle-même du degré auquel ces instances se sont avérées fiables ou trompeuses. ${ }^{37}$

32 Au crime et à l'énigme, anomalies premières du réel, s'ajoutent un lot de discordances et de réfutations. Mais si ces dernières entravent l'élucidation, elles y contribuent aussi dans la mesure où elles pourraient servir, comme l'investigation, à "mettre en question la réalité apparente, pour atteindre une réalité qui serait à la fois plus cachée [...] et plus réelle" ${ }^{38}$. Quelle entité, dès lors, se montre plus pertinente dans le sens qu'elle donne aux événements? L'indétermination demeure, les récits cultivant ce que Philippe Corcuff appelle "l'ironie sombre à l'égard de soi [...] et des autres, voire le cynisme et un relativisme immodéré (dans le tout se vaut)" ${ }^{\prime 3}$. Ce n'est pas un hasard s'ils se retrouvent en conséquence dominés par un éventail de procédés rhétoriques au sein duquel la négativité constitue le matériau sémantique privilégié. À l'appui, par exemple, d'antiphrases significatives, ces voix narratives chahutées balancent à demimots leurs vérités rongées par l'imposture.

Hôpital Van Horne. Service de neuropsychiatrie du docteur Manfred Zymanski. Pavillon 4. Aile sud. Un endroit accueillant. Si on aime la blancheur, l'odeur de l'hydroxyéthyl industriel mélangée à l'iode et au clou de girofle. Si on aime les gestes trop lents, les regards trop fixes des pensionnaires qui, pour la plupart, déambulent en toute liberté dans les jardins ensoleillés. Simplement retenus par des chaînes, des barreaux plus puissants que ceux de la prison la plus moderne. Des entraves qui s'appellent Dilaudid 4, Dopa L, Quaalud ou Lignocaïne. (An:37)

Les éléments modalisateurs et le lexique clinique intensif illustrent de façon paradoxale, jusqu'au détachement le plus glacial, l'absence de sensibilité du narrateurpersonnage (qui pourtant semble s'émouvoir de la froideur des lieux). Par ces entremises, la narration se fait le chantre d'un double jeu énonciatif, cas dans lequel il n'y a pas, selon le linguiste Alain Berrendonner, 
de meilleure hypothèse possible que l'interprétation paradoxale. Le contenu verbal énoncé inclut des commentaires méta-énonciatifs [...] tels que leur propre énonciation ne peut y être soustraite. Et comme ils contredisent certains de ses aspects posturaux, il devient impossible de parvenir à une interprétation cohérente. Il ne reste alors d'autre issue, pour [...] sauver la présomption de pertinence, que de supposer un énonciateur duplice. ${ }^{40}$

\section{Croire en rien, sauf en le roman noir?}

Si le roman noir français du XXI ${ }^{e}$ siècle multiplie les polyphonies, les contradictions et les "discours incompatibles à propos d'un même objet", il le fait toujours "à des fins incriminatoires" ${ }^{41}$. Sans doute ces indices contribuent-ils à un nouveau marquage idéologique du genre caractérisé par la destitution autoritaire des uns et la contestation de la véridicité des autres. Une dénégation qui s'attaque aussi à la signification inhérente au réel et à toute tentative d'ordonnancement de celui-ci.

Sur ce point, l'irrésolution à l'œuvre amène à considérer, au-delà du "faire semblant" du "narrateur duplice" évoqué par Alain Berrendonner, l'ultra-scepticisme et l'ultradésordre établi que mettent définitivement en place ces récits. À l'instar de ce qu'écrit Luc Boltanski, personne ici "ne croit plus aveuglément ce que disent les experts" (narrateur et personnages dans le cas présent, représentants faillibles d'une autorité d'énonciation et d'élucidation), "ce qui est préjudiciable à la vérité vraie" ${ }^{42}$. En filigrane des intrigues et des relations déployées apparaît bel et bien ce que souligne le sociologue : “tout le monde croit à n'importe quoi et personne ne croit plus à rien. C'est le retour du nihilisme" ${ }^{43}$.

\section{NOTES DE FIN}

1. Genre apparu à la fin des années 1970 sous l'impulsion du romancier Jean-Patrick Manchette. Une production qui "s'inscrit en rupture à la fois avec le modèle de la littérature policière d'énigme symbolisée par Agatha Christie (en préférant à la question Qui a tué ? celle du Pourquoi ? des événements et de leur issue) avec les sujets [...] et les styles de prédilection des anciens du polar français." (Annie Collovald et Erik Neveu, "Le néo-polar. Du gauchisme politique au gauchisme littéraire”, Sociétés \& Représentations n¹1, Paris, Publications de la Sorbonne, 2001, p. 77.)

2. Texte d'introduction du dossier "Le polar entre critique sociale et désenchantement", Mouvements, vol. 15-16, n³, 2001, p. 6.

3. Lire à ce sujet: Stéphane Ledien,"Enchantés d'être désenchantés... ou pas”, Le Crachoir de Flaubert, url : <http://www.lecrachoirdeflaubert.ulaval.ca/2019/03/enchantes-detredesenchantes-ou-pas/>.

4. Aurélien Masson, "Tant qu'il y a du noir, il y a de l'espoir”, dans Franck Lhomeau, Alban Cerisier (dirs), C'est l'histoire de la Série Noire: 1945-2015, Paris, Gallimard, 2015, <Albums Beaux Livres>, p. 147.

5. Baptiste Liger, “Sans vieux, ni maître”, Lire, juin 2009, p. 33. 
6. Ibid., p. 32.

7. DOA, Dominique Manotti, L'honorable société, Paris, Gallimard, 2011, <Série Noire> ; dorénavant HS.

8. Dominique Manotti, Bien connu des services de police, Paris, Gallimard, 2010, <Série Noire>.

9. Elsa Marpeau, Les yeux des morts, Paris, Gallimard, 2010, <Série Noire>.

10. "La plupart [de ces] auteurs refusent [...] de se considérer d'une école ou d'un courant, tout en reconnaissant une proximité entre eux." (Baptiste Liger, op. cit., p. 34.)

11. Dominique Manotti, “Roman noir”, Le Mouvement Social, n²19-220, 2007, p. 108.

12. Juliette Rennes, Simon Susen, "La fragilité de la réalité. Entretien avec Luc Boltanski", Mouvements, n64, 2010, p. 163.

13. Ibid.

14. Dominique Manotti, “Roman noir”, op. cit., p. 109.

15. Ibid.

16. Baptiste Liger, op. cit., p. 33.

17. Elsa Marpeau, Black Blocs, Paris, Gallimard, 2012, <Série Noire>. Dorénavant BB.

18. Jérôme Leroy, Le Bloc, Paris, Gallimard, 2011, <Série Noire>. Dorénavant LB.

19. Jérôme Leroy, L'ange gardien, Paris, Gallimard, 2014, <Série Noire>. Dorénavant AG.

20. Antoine Chainas, Pur, Paris, Gallimard, 2013, <Série Noire>.

21. Baptiste Liger, op. cit., p. 33.

22. Antoine Chainas, Anaisthêsia, Gallimard, Paris, 2011 [2009], <Folio Policier>. Dorénavant An.

23. Ibid., extrait de la dernière de couverture du roman.

24. Elsa Marpeau, Et ils oublieront la colère, Paris, Gallimard, 2015, <Série Noire>. Dorénavant Co.

25. Dominique Manotti, Or noir, Paris, Gallimard, 2015, <Série Noire>.

26. Déjà héros des romans Sombre sentier, À nos chevaux et Kop, respectivement parus pour la première fois en 1995, 1997 et 1998 aux éditions Rivages, dans la collection Rivages/Noir. Or Noir se passe en revanche en 1973, au tout début, donc, de la carrière du commissaire.

27. On y retrouve des personnages présentés dans le non moins volumineux Citoyens clandestins (Paris, Gallimard, 2015 [2007], <Folio Policier>).

28. DOA, Pukhtu : primo, Paris, Gallimard, 2015, <Série Noire>. Dorénavant PP.

29. "Je ne dénonce rien. Je ne m'inscris pas dans les postures de dénonciation qui sont une tradition dans le roman noir français, et de façon générale dans le roman français. Mon optique de réalisme poussé est confondue, à tort, avec la volonté de dénoncer quelque chose, alors qu'il s'agit juste pour moi d'une matière première" (Anne Brigaudeau, "Comment s'écrit un polar ? Rencontre avec DOA, qui dénonce l'ostracisation du roman noir", France Info : Culture, url : <https:// www.francetvinfo.fr/culture/livres/roman/comment-s-039-ecrit-un-polar-rencontre-avec-doaqui-denonce-quot-1-039-ostracisation-du-roman-noirquot_3365821.html>).

30. "Je dirai d'un narrateur qu'il est digne de confiance quand il parle ou agit en accord avec les valeurs de l'œuvre, et je le dirai indigne de confiance dans le cas contraire." (Wayne C. Booth, "Distance et point de vue", dans Gérard Genette, Tzvetan Todorov (dirs), Poétique du récit, Paris, Éditions du Seuil, 1977, p. 105.)

31. “Je n'écris pas des livres pour étaler mes préférences politiques. Elles ne regardent que moi et sont bien éloignées des thèmes que j'aborde." (Antoine Chainas, cité par Baptiste Liger, op. cit., p. 33)

32. Frank Wagner, "Narrateur non fiable / Unreliable Narrator", Glossaire du Réseau des Narratologues Francophones, url : <https://wp.unil.ch/narratologie/2019/11/narrateur-non-fiableunreliable-narrator/>.

33. Ibid.

34. Franck Frommer, Jean-Patrick Manchette - Le récit d'un engagement manqué, Paris, Kimé, 2003, p. 10. 
35. Pierre Schoentjes, Poétique de l'ironie, Paris, Éditions du Seuil, 2001,<Points/Essais-Inédits>, p. 115.

36. Ibid., p. 118.

37. Luc Boltanski, Énigmes et complots : une enquête à propos d'enquêtes, Paris, Gallimard, 2012, <NRF Essais>, p. 292.

38. Ibid., p. 61.

39. Philippe Corcuff, "Désenchantement et éthiques du polar", Mouvements 3/2001, op. cit., p. 103.

40. Alain Berrendonner, "Portrait de l'énonciateur en faux naïf", Semen, url: http:// journals.openedition.org/semen/2400.

41. Ibid.

42. Luc Boltanski, op. cit., p. 276.

43. Ibid.

\section{RÉSUMÉS}

Dans des romans noirs ambigus à l'extrême signés par des écrivains tels que DOA (Pukhtu), Antoine Chainas (Anaisthêsia, Pur), Jérôme Leroy (Le Bloc, L'ange gardien), Dominique Manotti (Bien connu des services de police, L'honorable société - coécrit avec DOA -, Or noir) et Elsa Marpeau (Black Blocs, Et ils oublieront la colère), le réalisme de la représentation se voit subverti au plus haut point. C'est avec une véhémence doublée d'une défiance confinant au nihilisme que les voix de ces romans prennent à partie la société et ses travers. En se mettant dans la peau d'activistes anarchistes (DOA \& Manotti, Marpeau), de policiers misanthropes (Chainas), de mercenaires ayant perdu toute attache idéologique (DOA), ou de fascistes s'éliminant entre eux au moment de l'accession au pouvoir d'un parti inspiré du Front National (Leroy), ces textes apparaissent comme une rupture et une réinvention génériques. Témoignant entre autres du chaos de l'après-11 Septembre, des ravages de la mondialisation et des crises identitaires et migratoires, ces romans mettent fin à une certaine tradition (celle des polars politisés de Jean-Bernard Pouy, Didier Daeninckx ou encore Patrick Raynal), en ne plaçant plus leurs narrateurs et personnages dans le camp de ceux qui "agissent bien". Au-delà d'une narration qui, quelque peu délétère, joue à cache-cache avec le bon sens moral, les récits proposent ainsi une peinture duale des rapports de méfiance entre différentes figures de pouvoir.

\section{INDEX}

Mots-clés : roman noir, conflits d'autorité, ironie, nihilisme, méfiance

\section{AUTEURS}

\section{STÉPHANE LEDIEN}

Université Laval 